\title{
Survivability in multi-domain optical networks using p-cycles
}

\author{
Hamza Drid · Samer Lahoud · Bernard Cousin · \\ Miklós Molnár
}

Received: 26 July 2009 / Accepted: 23 August 2009

(C) Springer Science+Business Media, LLC 2009

\begin{abstract}
Survivability is becoming an important issue in optical networks due to the huge bandwidth offered by optical technology. Many works have studied network survivability. The majority of these works are destined for single-domain networks. In this work, we address the survivability of multi-domain optical networks. This paper provides a classification of the existing protection solutions proposed for multi-domain networks and analyses their advantages and limitations. We propose a new solution for multi-domain optical networks based on p-cycles (pre-configured cycles). For scalability and security reasons, we also propose a topology aggregation model adapted to p-cycle computations. This aggregation model allows our proposed solution to find a trade-off between two competing goals: efficient use of backup resources and short running time. Simulation results show that the proposed solution is a good trade-off between resource utilization and running time compared to existing solutions.
\end{abstract}

Keywords Multi-domain - Survivability · p-cycle . Topology aggregation $\cdot$ Optical network

\author{
H. Drid $(\varangle) \cdot$ S. Lahoud · B. Cousin \\ University of Rennes I - IRISA, Campus universitaire de Beaulieu, \\ Rennes 35042, France \\ e-mail: hdrid@irisa.fr \\ S. Lahoud \\ e-mail: slahoud@irisa.fr \\ B. Cousin \\ e-mail: bcousin@irisa.fr \\ M. Molnár \\ INSA of Rennes - IRISA, Dpartement informatique, \\ Rennes 35043, France \\ e-mail:molnar@irisa.fr
}

\section{Introduction}

Survivability in optical networks is an important issue due to the huge bandwidth offered by optical technology. Survivability means that the network has the ability to maintain an acceptable level of service even after failures within the network. Therefore, operators expect survivability to be incorporated in the design and engineering of optical networks. This requirement becomes more critical as the size of networks increases.

Network survivability mechanisms may be classified according to two main categories: restoration and protection. Protection is a pro-active approach in which the backup path is reserved at the same time with the working path setup. Restoration is a reactive approach in which a backup path is computed and established after a failure in the working path. Protection is the key mechanism used to ensure survivability in optical networks because it guarantees full recovery whereas restoration may not offer the same guarantee if resources are not available. Since the single-fiber-link mode of failure is dominant in WDM optical networks, several protection mechanisms against a single-link failure have been proposed in the literature. These mechanisms are typically destined for single-domain protection, because they assume that each node in the network has a complete vision of the physical topology of the network. Such an assumption is not realistic in the case of large networks, such as multi-domain networks.

A multi-domain network is a network composed of several single-domain networks, interconnected by inter-domain links. Each domain can be regarded as an independent network that has its own local rules of operation and management to provide services [1]. Due to scalability constraints and domain management policies, the internal topological details of a domain are usually not shared externally. As a result, no node in a multi-domain network can have complete 
information on a multi-domain network. For instance, complete information corresponds to the detailed states of wavelength usage on each link of a multi-domain optical network. Thus, protection of multi-domain networks is more difficult than that of single-domain networks.

Solutions proposed for the protection problem in multidomain optical networks can generally be classified into two classes. In the first class [2-6], for each connection demand two link-disjointed paths (working and backup paths) from source node to destination node are computed. The drawback of this class of solutions is that the recovery time is very long. This is a consequence of the fact that the backup path is routed across the entire network. Moreover, the nodes performing the restoration are the source and destination nodes, and the failures should be notified to all nodes on the working path. Thus, this protection requires a large number of resource management operations and is not acceptable if the domains belong to competitive operators [7].

In the second class of solutions, each domain [1,7-9] protects the segment of the working path that crosses it. Consequently, the end-to-end primary lightpath is protected. This approach is rather scalable since each network operator protects its domain links independently of the links of the other domains. But the difficulty consists in the protection of the inter-domain links that are not protected by any domain.

In order to overcome the drawbacks mentioned above, we propose to use p-cycles (pre-configured cycles) to protect multi-domain optical networks and to improve the existing solution proposed by Szigeti et al. in [13]. An important property of p-cycles is that the cycles are fully pre-configured with pre-planned spare capacity and, when a link fails, only the two end nodes of the failed link need to perform the recovery actions, and no switching actions are required at any intermediate nodes of the cycles. This property significantly improves the p-cycles recovery time. For scalability and confidentiality (confidentiality of topological and operational parameters) reasons, we propose a new topology aggregation model to represent each domain in a compact manner. Several topology aggregation models are available in the literature [10-12]. However, the model that we propose is designed for p-cycle computation.

In the following section, we give an overview of protection using p-cycles. In addition, we present a p-cycle based protection scheme suitable for multi-domain optical networks [13] and discuss its limitation. In Sects. 3 and 4, we detail our solution and show the performance results. In Sect. 5, we conclude the paper.

\section{Multi-domain protection using p-cycles}

Single domain network protection based on p-cycles has been extended for multi-domain network protection. The use of

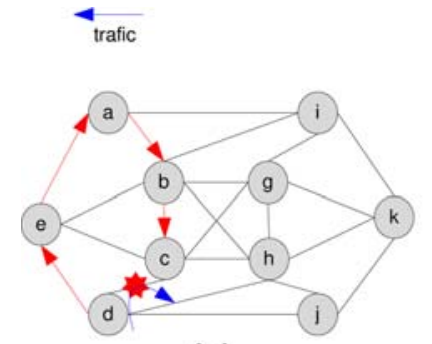

(a)

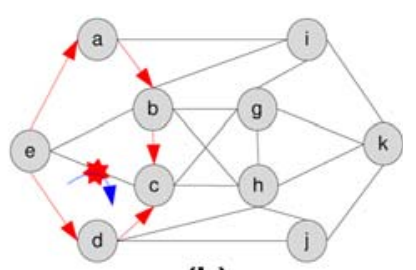

(b)
Fig. 1 p-Cycle protection

p-cycles for multi-domain network protection is proposed and evaluated in reference [13]. In this section, we explain the concept of p-cycles and their advantages. In addition, we describe the solution proposed in reference [13] and its limitations.

\section{1 p-Cycles}

A p-cycle is an efficient approach for protecting working capacities in WDM networks, because p-cycles can achieve a fast restoration time like ring protection and a high efficiency capacity like mesh protection [14]. p-Cycle achieves fast restoration times since only the nodes adjacent to the failure perform protection switching. The high capacity efficiency is due to the fact that a p-cycle can provide protection not only for on-cycle links but also for straddling links without requiring any additional spare capacity. A straddling link is a link which does not belong to the p-cycle but whose end-nodes are both on the p-cycle. This property effectively reduces the required protection capacities.

Figure 1 depicts an example that illustrates p-cycle protection. In Fig. 1a, nodes a-b-c-d-e-a form a p-cycle with one unit of spare capacity (which means one wavelength on each on-cycle link). When the on-cycle link (d-c) fails as shown in Fig. 1a, the p-cycle provides one protection path (d-e-a-b-c). In Fig. 1b, an example of a straddling link failure is shown. When the straddling link $(\mathrm{e}-\mathrm{c})$ breaks, the p-cycle can protect two working wavelengths on this link by providing respectively two alternate paths $(\mathrm{e}-\mathrm{d}-\mathrm{c})$ and $(\mathrm{e}-\mathrm{a}-\mathrm{b}-\mathrm{c})$.

The efficiency of a p-cycle is inversely proportional to its redundancy. The redundancy [15] of a cycle is defined as the ratio between the spare capacity (number of wavelengths used by the cycle) and the working capacity of the cycle (number of wavelengths protected by the cycle on its oncycle and straddling links). A p-cycle with high efficiency is a p-cycle that has a small value of redundancy, in other words it protects more working wavelengths using fewer spare wavelengths. We define the redundancy of a p-cycle $i$ as follows:

$\Re(i)=\sum_{\forall j \in E} P_{i, j} / \sum_{\forall j \in E} X_{i, j}$ 


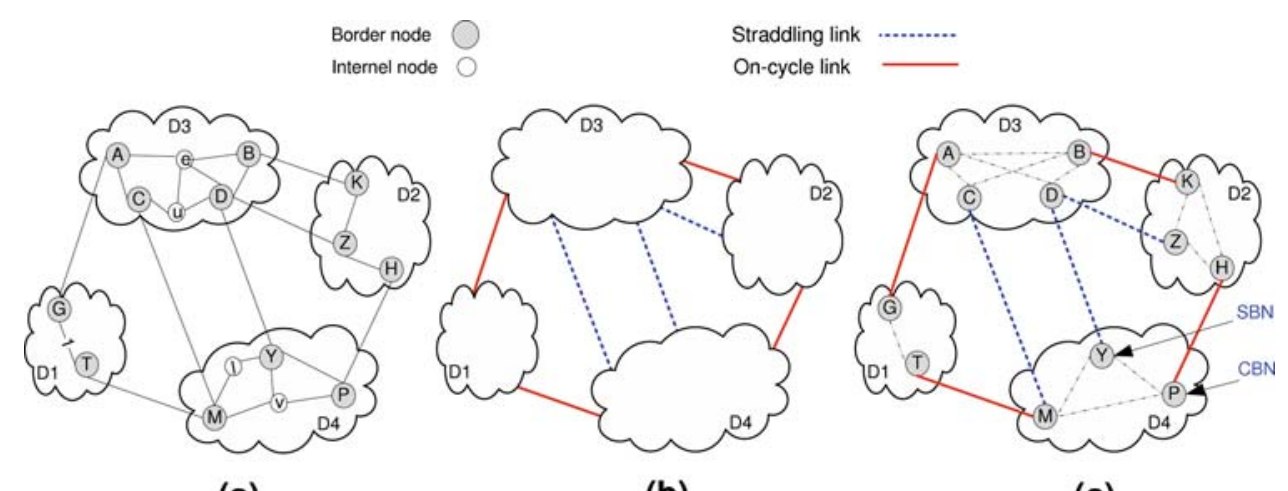

(a)

(b)

(c)

Fig. 2 Multi-domain protection using p-cycles

Let $E$ be the set of network links. $P_{i, j}$ is the number of working capacity required on link $j$ to construct p-cycle $i . X_{i, j}$ is the number of wavelengths that p-cycle $i$ protects on link $j$. $X_{i, j}$ takes the value 2 if $j$ is a straddling link of p-cycle $i$, and it takes the value 1 if $j$ is an on-cycle link of p-cycle $i$.

The redundancy $R$ of the final protection solution is computed as the ratio between the total spare capacity used for protection design and the protected working capacity in the network.

$\Re=\sum_{\forall j \in E} S_{j} / \sum_{\forall j \in E} W_{j}$

$S_{j}$ and $W_{j}$ are the numbers of spare and working wavelength capacity on link $j$.

\section{2 p-Cycle based protection schemes for multi-domain networks}

In reference [13], the authors have proposed a solution for a multi-domain network protection based on p-cycles. The main goal of this solution is to protect the inter-domain links.

This solution proceeds in three steps. In the first step, the set of p-cycles protecting the inter-domain links is computed. This set of p-cycles is designed over a single-node virtual topology (see Fig. 2b), in which each domain is represented by a single virtual node. The second step consists of determining, for each p-cycle calculated in the previous step, the border nodes to which its links (the on-cycle and straddling links) are connected. The border nodes connected to the on-cycle inter-domain link of a p-cycle are called on-cycle border nodes (denoted by cbn) and those connected to a straddling inter-domain links are called straddling border nodes (denoted by sbn). Moreover, in this step, the end nodes of inter-domain on-cycle and straddling links need to be connected internally to ensure the continuity of the p-cycle. The internal links connecting the cbn and the sbn nodes are virtual (see Fig. 2c). During the last step, each internal virtual link calculated in the second step is translated into a physi- cal path. Note that the calculated p-cycles bypass the intradomain links without the possibility of protecting them.

This approach has some drawbacks related to the quality of the set of p-cycles protecting the inter-domain links. At first, the p-cycles protecting the inter-domain links are computed over a single-virtual-node topology. This virtual topology reduces a domain with multiple links and nodes to a single virtual node. Such a representation hides all topological information describing the domain. Consequently, the real cost of a p-cycle on the physical topology is unknown. The second drawback, which we can point to in this solution, is due to the fact that the set of p-cycles obtained is designed only to protect the inter-domain links. Nevertheless, there exist communications within each domain which can be protected by the existing p-cycles, without using additional resources. The communication links which can be protected freely are those passing through the intra-domain links crossed by the existing p-cycles.

\section{Our proposed solution}

After presenting the existing solution [13] and its major drawbacks, we develop in this section our motivation and objectives in developing a new approach for multi-domain protection using p-cycles. We start by a thorough analysis of the limitations presented in the previous section. Then we introduce the main guidelines of our solution that enable us to overcome these limitations.

\subsection{Motivation}

As mentioned previously, the solution suggested in reference [13] presents some disadvantages that strongly affect the quality of the set of p-cycles protecting the network (for example, the redundancy of the obtained p-cycles is very high). These disadvantages are mainly due to the lack of topological information for each domain. The following exam- 


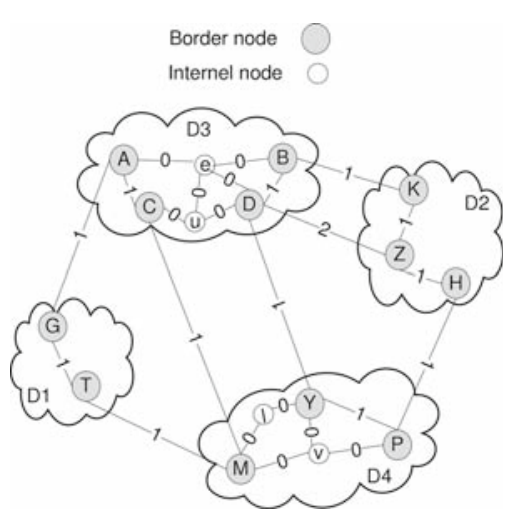

Fig. 3 Multi-domain optical networks

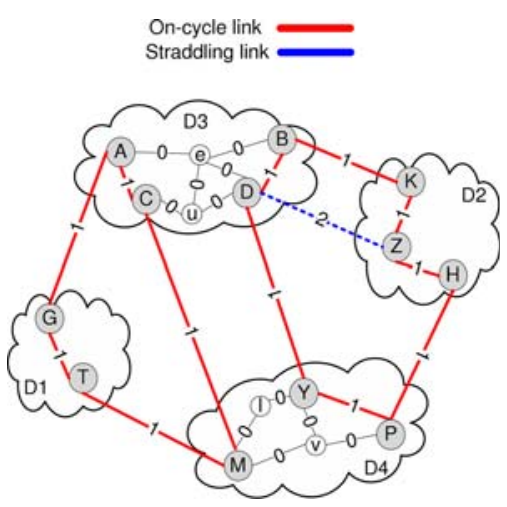

Fig. 4 Computed p-cycles with internal topological knowledge

ple shows how the lack of topological information for each domain affects the quality of p-cycles during the computation of p-cycles.

In this example, we calculate the optimal set of p-cycles (optimal in terms of redundancy) for two different topologies of the same multi-domain network (shown in Fig. 3). In the first topology, all domains (D1 to D4) are represented by their physical topology as shown in Fig. 3.

In the second topology, each domain is viewed as a single node as shown in Fig. 5a. The optimal set of p-cycles obtained for these two topologies are different. In the first topology, the solution consists of two p-cycles (A-C-M-TG-A) and (D-B-K-Z-H-P-Y-D) as shown in Fig. 4. In the second topology, the solution is composed of one p-cycle which traverses D1, D2, D3, D4 as shown in Fig. 5a. To evaluate the efficiency, we calculate the redundancy of each solution. The redundancy obtained by the first solution is: $\Re=$ $12 / 14=85.65 \%$.

To calculate the real redundancy of p-cycles computed on the single-virtual-node topology, we need to project the p-cycles in the physical topology as shown in Fig. 5b. After the projection of p-cycles on the real topology, the redundancy obtained is: $\Re=14 / 14=100 \%$. We note that the redundancy obtained by the first solution is better than that obtained by the latter one. This difference is due to the fact
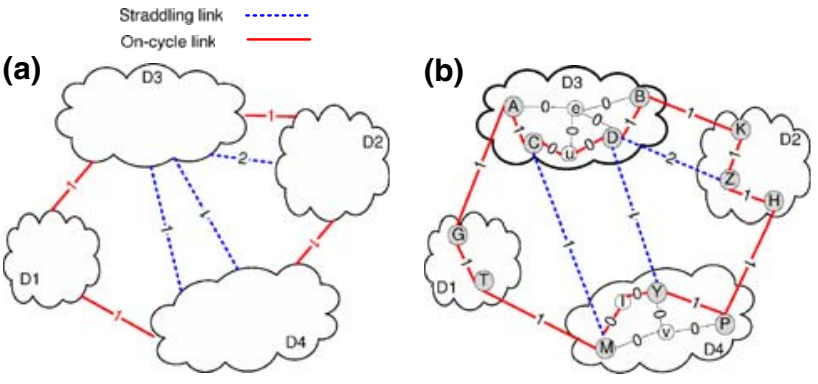

Fig. 5 Computed p-cycles with single-virtual-node domains

that the first solution takes into account the internal topology of each domain during the computation of p-cycles. To overcome these problems, the internal topology of each domain should be taken into account during the computation of p-cycles. However, as the size of a multidomain network is very large, the computation of the set of p-cycles becomes intractable as soon as we take into account all the details of the internal topology of each domain. Moreover, the network operators for the various domains would be very reluctant to distribute the data of their internal network topology. One solution to deal with this scalability problem is to represent the internal topology of each domain in a compact and abstract manner. This process is known as topology aggregation.

The basic idea of our proposal consists of describing the internal topology of each domain in a compact manner by using a topology aggregation model. This enables us to construct a new reduced multi-domain topology, on which we calculate the set of p-cycles protecting the network. As a result, the calculation of the p-cycles is accelerated while the scalability and confidentiality constraints are satisfied. Moreover the quality of the p-cycles is improved for the following reasons:

1. During the calculation of p-cycles, we have more information about the physical topology of each domain. This solves the first disadvantage of the proposal of reference [13].

2. Each calculated p-cycle may protect some working capacities in the traversed domains. This overcomes the second limitation of the proposal of reference [13].

Each calculated p-cycle contains a set of virtual links inside each of the traversed domains. Each virtual link is further translated into a light-path.

\subsection{A novel topology aggregation model}

The aggregation model that we propose in this study aims to represent the network topology and its working capacity in a compact manner. This model is also adapted to p-cycle 


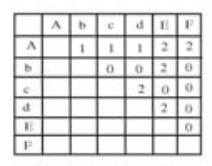

(a) Demands matrix

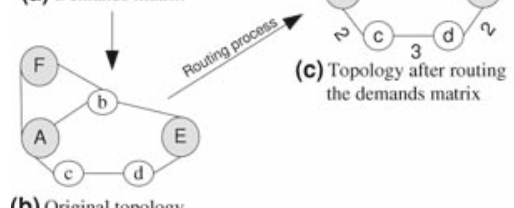

(b) Original topology
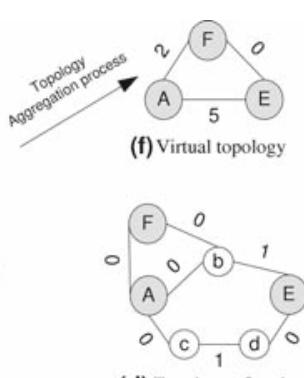

(d) Topology after the aggregation
Fig. 6 Topology aggregation model

computations. We transform the physical topology of each domain into a simple virtual topology.

The topology obtained after aggregation is composed of all border nodes, which are connected by virtual links. The virtual topology has the following characteristics:

- Each pair of border nodes within a domain is connected by a virtual link. A virtual link connecting a pair of border nodes corresponds to the set of primary lightpaths interconnecting these nodes in the physical topology (see Fig. 6c).

- An integer is associated with each virtual link indicating the number of primary lightpaths existing between the two border nodes.

- Two virtual links have to be physically disjointed, which means that the light-paths connecting one pair of border nodes must be disjointed from all the lightpaths between any other pair of border nodes.

Figure 6 describes an example that shows how our aggregation model generates a virtual topology. Figure $6 \mathrm{c}$ describes a network with six nodes, of which three (A, E, F) are border nodes. For each link, an integer value indicating the working capacity of the link is associated. This working capacity is obtained by routing the demand matrix.

In Fig. 6e, the virtual topology obtained from the original topology by aggregation is shown. The virtual topology is only composed of border nodes and virtual links.

The capacities of the links (A-E), (A-F), and (F-E) in the virtual topology indicate the number of disjointed lightpaths existing between each pair of border nodes. The virtual link (A-E) corresponds, for instance, to the following lightpaths: two lightpaths using the path $(A \rightarrow c \rightarrow d \rightarrow E)$ and three lightpaths using $(A \rightarrow b \rightarrow E)$. The virtual link A-F corresponds, for instance, to two lightpaths using fiber A-F.

We also illustrate in Fig. 6d the remaining working capacity that could not be aggregated into the virtual topology. After building the virtual topology of each domain, the set of

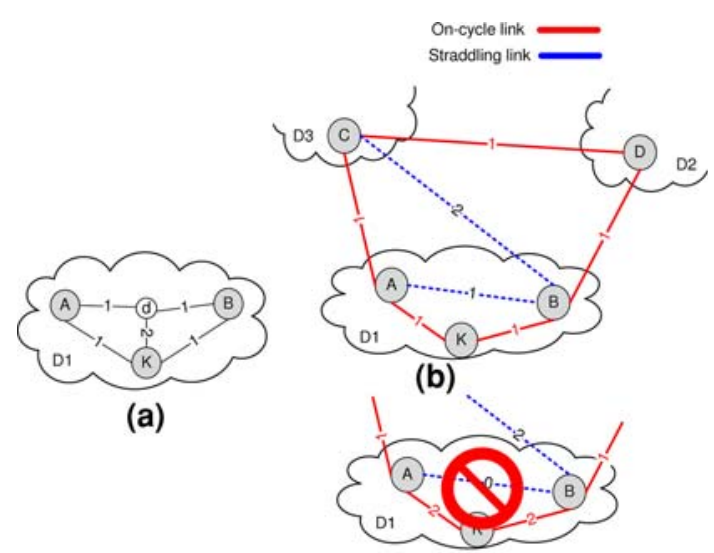

(c)

Fig. 7 Proposed model of topology aggregation

p-cycles is computed over the virtual topology of the multidomain network.

Note: The virtual topology of the multi-domain network is composed of the virtual domains, which are interconnected by the inter-domain links.

One of the important properties of a p-cycle is that its links must be physically disjointed. This property ensures that the traffic crossing a link that is potentially failing can be re-routed to the other links of the p-cycle without any interruption of the traffic. For this reason, the virtual links of the topology have to be physically disjointed. If the computed virtual topology does not have this property, the failed link and the links that will protect it, could share physical links. Consequently, when a failure occurs, several virtual links of different p-cycles could fail at the same time and the traffic would not be recovered.

With the help of the following example, we illustrate the aforementioned disjointness constraint. In Fig. 7b, A-K-B$\mathrm{D}-\mathrm{C}$ is a p-cycle composed of five links and goes through three different domains D1, D2, and D3. When the on-cycle link $\mathrm{A}-\mathrm{K}$ fails, the p-cycle provides one protection path (A-C-D-B-K) to protect one wavelength of the virtual link A-K.

Figure $7 \mathrm{~b}$, c illustrates two possible virtual topologies that we can obtain from the original topology shown in Fig. 7a. In the first virtual topology, let us suppose that all virtual links are physically disjointed. For instance, the virtual link A-K ( $\mathrm{K}-\mathrm{B}$, and $\mathrm{A}-\mathrm{B}$, respectively) corresponds to the lightpath $\mathrm{A}-\mathrm{K}(\mathrm{K}-\mathrm{B}, \mathrm{A}-\mathrm{d}-\mathrm{B}$, respectively) in the original topology. In this case, the traffic can be recovered in domain D1.

In the second virtual topology shown in Fig. 7c, the virtual links $\mathrm{A}-\mathrm{K}$ (which corresponds to the lightpaths $\mathrm{A}-\mathrm{d}-\mathrm{K}$ and $\mathrm{A}-\mathrm{K}$ ) and $\mathrm{K}-\mathrm{B}$ (which corresponds to the lightpaths $\mathrm{K}-\mathrm{d}-\mathrm{B}$ and $\mathrm{K}-\mathrm{B}$ ) share the physical link $\mathrm{d}-\mathrm{K}$. Consequently, if this link fails, the two virtual links fail at the same time and the traffic cannot be re-routed. 


\subsection{ILP model for topology aggregation}

In this section, we present an Integer Linear Programming (ILP) formulation for our topology aggregation. We start by introducing some notations for our model. A multi-domain networks composed of $M$ connected domains can be represented by a graph $G=\left(O_{i}, L\right), i=1,2, \ldots, M$, where $O_{i}$ and $L$ represent, respectively, the graph of optical domain number $i$ and the set of inter-domain links.

We model an optical domain $O_{i}$ as an undirected graph $O_{i}=\left(V_{i}^{b}, V_{i}^{i}, E_{i}\right)$, where $V_{i}^{b}=\left\{v_{i, 1}^{b}, \ldots, v_{i, b_{i}}^{b}\right\}, V_{i}^{i}=$ $\left\{v_{i, 1}^{i}, \ldots, v_{i, n_{i}}^{i}\right\}$ and $E_{i}$ represents the set of border nodes, the set of interior nodes and the set of intra-domain links in domain $i$, respectively. In our model, an intra-domain link connects nodes of the same domain. We also consider that the i-th domain has $n_{i}$ interior nodes and $b_{i}$ border nodes.

Each link $e$ in $E_{i}$ supports $w_{e}$ working wavelength channels. This working capacity is obtained by routing the demand matrix. We assume that the demand matrix, consisting of the number of wavelength channels to be established between each node pair, is given.

The ILP formulation determines the maximum number of working lightpaths connecting any pair of border nodes satisfying constraints (4) and (5). Constraint (4) ensures that the number of lightpaths crossing link e should not exceed the number of the primary wavelengths available on this link. Constraint (5) guarantees that the primary lightpaths interconnecting a border node pair are link disjointed from all lightpaths connecting any other border node pair. In the output of the ILP, the number of physical lightpaths between two border nodes defines the capacity of the virtual link connecting these two nodes in the virtual topology.

The objective function is:

Maximize $\sum_{d \in D_{i}} \sum_{p \in P(d)} h_{d, p}$

Subject to:

$$
\begin{aligned}
& \sum_{d \in D_{i}} \sum_{p \in P(d)} a_{e, d, p} h_{d, p} \leq w_{e} \quad \forall e \in E_{i} \\
& \sum_{d \in D_{i}} Y_{e, d} \leq 1 \quad \forall e \in E_{i}
\end{aligned}
$$

Let $D_{i}=\left\{\left(v_{i, i}^{b}, v_{i, j}^{b}\right) \backslash \forall j>i, 1 \leq i, j \leq b_{i}\right\}$ be the set of border node pairs in the domain $i . P(d)$ indicates the set of all paths connecting the pair of border nodes $d$, where $d \in D_{i} . h_{d, p}$ is an integer variable that indicated the number of lightpaths connecting the pair of border nodes $d$.

$\forall e \in E_{i}, \forall d \in D_{i}, \quad Y_{e, d}= \begin{cases}0 & \text { if } \sum_{p} a_{e, d, p} h_{d, p}=0 \\ 1 & \text { if } \sum_{p} a_{e, d, p} h_{d, p} \leq 0\end{cases}$
$Y_{e, d}$ is a binary variable that indicates whether one of the lightpaths connecting the border node pair $d$ crosses link $e$.

$\forall d \in D_{i}, \forall p \in P(d), \forall e \in E_{i}, \quad a_{e, d, p}= \begin{cases}0 & \text { if } e \notin p \\ 1 & \text { if } e \in p\end{cases}$

$a_{e, d, p}$ is a binary constant which indicates if lightpath $p$ of border node pair $d$ crosses link $e$.

\section{Results and analysis}

The performance of our solution is evaluated in this section. Simulation experiments are carried out using three widely used topologies taken from references [1] and [16] and characterized in Table 1. The first two topologies contain three different domains, while the last contains four domains. Between two different domains, the node-pair is interconnected by a bi-directional fiber link. Each fiber link is assumed to have 128 wavelengths, and each network node is assumed to have the wavelength conversion capacity.

All simulations were run on a DELL Quadri Dual Core Xeon processor with $4 \mathrm{~GB}$ of RAM. MATLAB is used to solve the ILP formulations.

The traffic demand is uniformly distributed among all source-destination pairs. Each demand requests one unit of capacity. The working capacities on the network links are obtained by routing each demand over the shortest path. Several experiments are performed for each topology network and the average values are presented as the final results.

The performance metrics that we have considered are:

Redundancy: Redundancy constitutes a major evaluation criterion for WDM network design. It gives an insight into the quality and relative cost of network protection. The redundancy enables to measure the protection efficiency: it denotes the ratio between the spare capacity (protection capacity) and the working capacity in the network.

Computational complexity: Computational complexity is an important factor when evaluating an algorithm. It is defined as the time it takes for an algorithm to find a solution. If an algorithm can achieve a near optimal solution with acceptable computational complexity, it will likely be a desirable solution to the problem.

Table 1 Topology characteristics

\begin{tabular}{llll}
\hline Characteristics $\backslash$ Topologies & US network & ARPANET & T3 \\
\hline Nodes & 15 & 20 & 44 \\
Links & 25 & 32 & 69 \\
Nodal degree & 3.33 & 3.2 & 3.13 \\
Demands & 128 & 174 & 490 \\
Working capacity & 245 & 457 & 1941 \\
\hline
\end{tabular}


Several scenarios are also considered to evaluate our solution:

Dedicated-backup: In the first scenario, we implement dedicated $1+1$ backup path protection. In dedicated backup path protection, both the working and the backup path are link disjointed. The resources along a backup path are uniquely reserved for only one working path. The complete topologies of the multi-domain networks are available for the calculation of the set of p-cycles.

Full-information: In this scenario, the p-cycle based protection is implemented. The complete topologies of the multidomain networks are available for the calculation of the set of p-cycles. The p-cycles protecting the multi-domain networks are computed using the exact solution proposed in [1]. This solution uses an ILP to find the optimal set of p-cycles in terms of redundancy.

Aggregated-topology: In the third scenario, the topologies of the multi-domain networks are transformed into a virtual topology by using our aggregation model. The set of p-cycles is computed on the virtual topology by using the same algorithm used in the previous scenario.

Note: The working capacity remaining in each domain after the aggregation of the original topology is also protected by p-cycles.

Single-node: In this scenario, we implement the solution proposed in [13] and described in the Sect. 2 of this paper. In this solution each domain is represented by a single node and the inter-domain and intra-domain links are independently protected using p-cycles.

The results summarized in Table 2 show the values of redundancy obtained in the different scenarios. Table 3 compares the running time taken by the different scenarios to compute the set of p-cycles protecting the network.

Table 2 Redundancy

\begin{tabular}{llll}
\hline $\begin{array}{l}\text { Solutions } \backslash \\
\text { Topologies }\end{array}$ & $\begin{array}{l}\text { US network } \\
(\%)\end{array}$ & $\begin{array}{l}\text { ARPANET } \\
(\%)\end{array}$ & T3 (\%) \\
\hline Dedicated-backup & 149 & 153 & 138.82 \\
Full-information & 93.65 & 92.14 & - \\
Aggregated-topology & 99.19 & 96.35 & 103.78 \\
Single-node & 146.86 & 165 & 150.88 \\
\hline
\end{tabular}

Table 3 Computational complexity

\begin{tabular}{llll}
\hline Solutions $\backslash$ Topologies & US network (s) & ARPANET (s) & T3 (s) \\
\hline Dedicated-backup & 1.25 & 2.04 & 13.04 \\
Full-information & 330648.09 & 1055633.75 & - \\
Aggregated-topology & 10.5 & 15.5 & 29.76 \\
Single-node & 1.43 & 4.56 & 8.68 \\
\hline
\end{tabular}

We can see in Table 2 that the value of redundancy obtained in the second scenario (Full-information) is better than that obtained by the others scenarios for the first two topologies. This remark is justified by the fact that when the complete topologies of multi-domain networks are considered during the p-cycle computation, the resource optimization obviously works better. However, the running time of this solution is very long and grows with the size of the network as shown in the Table 3. We also note that the Full-information solution cannot find a result for the third topology.

This is due to the large size of this topology and the large number of working wavelengths in the network. This was part of our motivation for proposing a new topology aggregation model and for adapting it to p-cycles in order to reduce the topological complexity. Consequently, the running time taken by our solution (Aggregated topology) is very short compared to that taken by the optimal Full-information solution.

The redundancy of our solution of aggregated topology is 5.54 and $4.21 \%$ higher than the redundancy of the Fullinformation solution, respectively, for the US and ARPANET test networks. The main reason for this behavior is that the working capacity remaining after the aggregation (see Fig. 6d) is protected independently of the aggregated working capacity.

We can also see that the state-of-the-art Single-node solution requires more resources than our solution to protect the working capacity. This difference is due to two factors:

1. During the calculation of p-cycles, our solution uses more information about the physical topology of each domain (this information comes from the virtual aggregated topologies).

2. In our solution, each calculated p-cycle may protect some working capacities in the traversed domains. This is not the case for the single-node proposal. Thus, we significantly improved the redundancy value for our proposed solution.

\section{Conclusion}

In this study, we addressed the protection of multi-domain optical networks. We started by classifying the proposed solutions and by discussing their drawbacks. We proposed a new solution for the survivability of multi-domain optical networks based on p-cycles. For scalability and security reasons, we also proposed a new topology aggregation model adapted to p-cycle computations. This aggregation model enabled our solution to improve the quality of the computed p-cycles.

Moreover, our solution protects primary lightpaths within each domain using the spare capacity reserved for the existing 
p-cycles protecting the inter-domain links. Thus it improves significantly the redundancy parameter.

Simulation results show that our solution is a good tradeoff between two competing goals: efficient usage of backup resources and short running time. This study is very encouraging and we intend to continue our investigations by using other constraints such as the size of p-cycles and integrating domain management policies.

\section{References}

[1] Guo, L.: LSSP: a novel local segment-shared protection for multidomain optical mesh networks. Comput. Commun. 30(8), 17941801 (2007)

[2] Akyamac, A., Sengupta, S., Labourdette, J., Chaudhuri, S., French, S.: Reliability in single domain vs. multi domain optical mesh networks. In: National Fiber Optic Engineers Conference, Dallas ,USA, pp. 240-249, Sept 2002

[3] Truon, D., Thiongane, B.: Dynamic routing for shared path protection in multidomain optical mesh networks. J. Opt. Netw. 5(1), 58-74 (2006)

[4] Liu, Q., Ko, M., Ghani, N., Gumaste, A.: Hierarchical routing in multi-domain optical networks. Comput. Commun. 30(1), 122-131 (2006)

[5] Larrabeiti, D., Romeral, R., Soto, I., Uruena, M., Cinkler, T., Szigeti, J., Tapolcai, J.: Multi-domain issues of resilience, In: J. Pareta (ed.), Int. Conf. Transparent Optical Networks, Barcelona, Spain, pp. 375-380, July (2005)

[6] Herzberg, M., Shleifer, F., Ring, R., Zolberg, O.: Applying OD cycles to multi-domain, multi-service survivable networks. In: 3rd EuroNGI Conference on the Next Generation Internet Networks, Trondheim, Norway, pp. 80-87, May 2007

[7] Xie, X., Sun, W., Hu, W., Wang, J.: A shared sub-path protection strategy in multi-domain optical networks. In: Optical Fiber Communication and Optoelectronics Conference, Shanghai, China, pp. 418-420, oct 2007

[8] Achille, A., Listanti, M., Monaco, U., Ricciato, F., Sharma, V.: Diverse inter-region path setup establishment. In: IETF InternetDraft, Draft Dachille Diverse Interregion Path Setup (2004)

[9] Huang, C., Messier, D.: A fast and scalable inter-domain MPLS protection mechanism. J. Commun. Netw. 6(1), 60-67 (2004)

[10] Awaebuch, B., Shavitt, Y.: Topology aggregation for directed graphs. IEEE/ACM Trans. Netw. 9(1), 82-90 (2001)

[11] Lee, W.: Minimum equivalent subspanner algorithms for topology aggregation. In: ATM networks, International Conference on ATM, Colmar, France, pp. 351-359, June 1999

[12] Bhutani, K.R., Battou, A., Khan, B.: Two approaches for aggregation of peer group topology in hierarchical PNNI networks. Int. J. Intell. Autom. Soft Comput., Autosoft Press, 6(2), 125-134 (2000)

[13] Szigeti, J., Romeral, R., Cinkler, T., Larrabeiti, D.: p-Cycle protection in multi-domain optical networks. Photon. Netw. Commun. 17(1), 35-47 (2009)

[14] Grover, W.D., Stamatelakis, D.: Cycle oriented distributed preconfiguration: ring-like speed with mesh-like capacity for selfplanning network restoration. In: International Conference on Communications, Georgia, USA, pp. 537-543, june 1998

[15] Drid, H., Cousin, B., Lahoud, S., Molnór, M.: Multi-criteria p-cycle network design. In: IEEE Conference on Local Computer Networks, Montreal, Canada, pp. 361-366, Oct 2008

[16] Yang, X., Ramamurthy, B.: Inter-domain dynamic routing in multi-layer optical transport networks. In: IEEE GLOBECOM, San francisco, USA, pp. 2623-2627 (2003)

\section{Author Biographies}

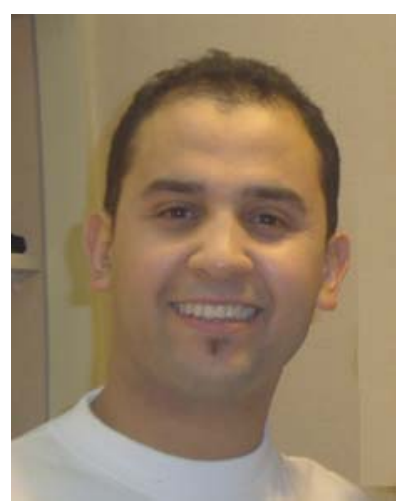

Hamza Drid received his Engineer Degree in Computer Science in 2004 from the University of Batna (Algeria), and a master degree from the university of Nancy 1 (France) in 2006. Currently, he is preparing for the $\mathrm{Ph} . \mathrm{D}$. degree in computer science at IRISA (Institut de recherche en informatique et systèmes aléatoires) laboratory in Rennes (France). His main research topics and interests include protection, MPLS, and high speed networks, resource optimization, routing, Survivability in multi-domain optical networks. He has been involved in many research programs at the national and the European level.

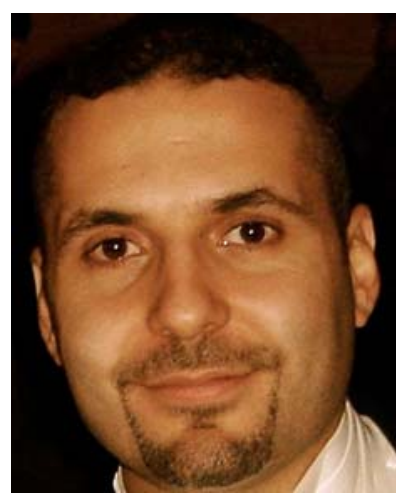

Samer Lahoud graduated from the Faculty of Engineering, Saint Joseph University, Beirut, in 2002. In 2006, he received the Ph.D. degree in Computer Science from Telecom Bretagne, Rennes. After his Ph.D. he spent 1 year with Alcatel-Lucent Bell Labs Europe working as a research engineer. Since 2007, he has been with the University of Rennes I, where he is working as assistant professor, and with IRISA of Rennes, where he is taking part in the research activities. His main research activity is in network design, combinatorial optimization and engineering algorithms for communication networks. He has been involved in many research programs at the national and the European level.

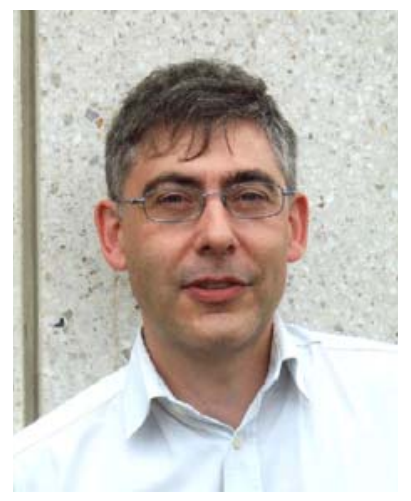

Bernard Cousin is a Professor of Computer Science at the University of Rennes 1 , France. Bernard Cousin received in 1987 his $\mathrm{Ph} . \mathrm{D}$. degree in computer science from the University of Paris 6. He is, currently, member of IRISA (a CNRS-University-INSA joint research laboratory in computing science located at Rennes). More specifically, he is at the head of a research group on networking. $\mathrm{He}$ is the co-author of a network technology book: "IPV6" (Fourth edition, O'Reilly, 2006) and has co-authored a few IETF drafts in the areas of Explicit Multicasting and Secure DNS. His research interests include all-optical networks, dependable networking, high speed networks, traffic engineering, multicast routing, network QoS management, network security and multimedia distributed applications. 


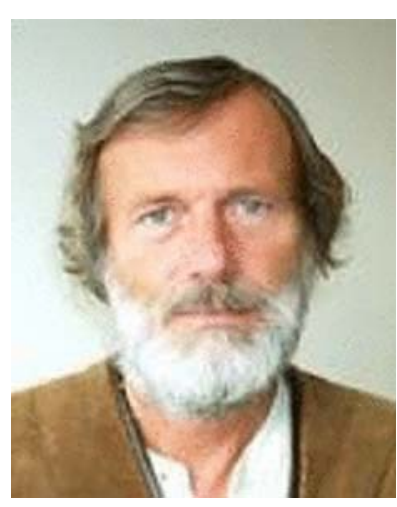

Miklós Molnár was graduated at the Faculty of Electrical Engineering, Technical University of Budapest in 1976. He received the Ph.D. degree in computer science from the University of Rennes 1 in 1992 and the HDR degree (accreditation to supervise research) in 2008. Since 1989, he has been with the INSA of Rennes, where he is working as associate professor, and with the research laboratory IRISA. His main research activity is in combinatorial optimization for communication networks: constrained optimization problems, QoS routing algorithms for unicast, incast and multicast communications, dependable communications, routing in optical and in wireless networks, energy aware protocols and optimizations. 